\title{
Don't be afraid of modulated structures
}

\author{
V Petricek ${ }^{1}$, M Dusek $^{2}$ \\ ${ }^{1}$ Institute of Physics, Czech Academy of Sciences, Prague, ${ }^{2}$ Institute of Physics, Czech Academy \\ of Sciences, Prague, Czech Republic \\ petricek@fzu.cz
}

The fact that the investigated crystalline substance could be modulated is a nightmare for many crystallographers. The main obstacle to understanding modulated structures is the need to use multidimensional space [1]. However, the way how we use the multidimensional space follows directly from the generalization of methods used for the study of standard structures. Any modulated structure can be easily detected from the diffraction pattern. Here, in addition to the dominant diffraction spots (main reflections), sharp spots called satellite reflections can be observed. Such reflections cannot be indexed by three indices related to the reciprocal lattice derived from the main reflections. Nevertheless, these spots are regularly distributed in space and can be indexed by adding one or more vectors to the reciprocal lattice. Standard programs supplied with modern diffractometers allow for indexing and subsequent processing of data of modulated structures, and users obtain data in a standard form, i.e. as reflection indices, integrated intensity, and their standard uncertainty. The only difference is that the number of indices is $(3+$ $\mathrm{d})$, where $\mathrm{d}$ is the number of the additional vectors. Standard structure analysis usually describes the structure by the positions of individual atoms, the occupation factors and isotropic or anisotropic atomic displacement parameters. The structure model of a modulated crystal is more complicated as some structure parameters must be described by modulation functions, which take a different form for each atom of the structure. In general, modulation function can be expanded into a Fourier series and then the expansion coefficients are new structure parameters. The initial expansion coefficients can be obtained automatically using the charge flipping program Superflip [2]. However, we have to decide how far to go in this generally infinite series. If we introduce too many parameters, we will get very good agreement with the measured data, but the solution may be wrong. Therefore, the complete automation of the modulated structure solution would not be beneficial. Moreover, it turns out that a modulation function can also have a discontinuous character, and then the development to the Fourier series would be tough. In such cases, more effective description of modulations can be achieved using special functions - crenel [3], saw-tooth and zig-zag, which on the other hand, cannot be introduced automatically. Modulation functions follow the symmetry of superspace described by superspace group [4]. The superspace symmetry can be revealed from the analysis of the symmetry of the diffraction pattern, and systematic absences. The sequence of steps is the same as in the classical crystallography: after determination of symmetry, we try to obtain a structure model, and in the next step, we verify the obtained structure parameters. To create the best model of a modulated structure, we have to inspect visually Fourier maps computed in $(3+\mathrm{d})$ space. Their special de Wolff sections allow comparing the modulation function resulting from the current structure model with the electron density (Fig 1). Individual atomic modulations are sometimes difficult to apply to bigger organic molecules because of the large number of refined parameters. Here we can use an assumption that modulation cannot significantly affect solid molecular fragments, and apply modulation functions directly to these fragments. Alternatively, we can apply restraints that ensure rigidity of the fragment. The confrontation of the model with the electron map is then performed using "animation" techniques. In cases where the purpose of structure analysis is just characterization of a newly prepared substance that does not have any significant physical properties in crystalline form. Therefore, in such cases, it is not out of the question trying to prepare an analogous structure that would not be modulated. However, the situation is entirely different in cases where the crystal has significant physical properties, and the modulations are directly related to them. Perhaps the best examples come from the field of magnetic structures. For magnetic structures, the concept of symmetry is extended by the symmetry of magnetic moments, which also includes the so-called time inversion [5], [6]. On the other hand, the main principles of the solution remain the same as for modulated structures. [1] de Wollf, P.M. (1974) Acta Cryst. A30, (1974) 777-785. [2] Palatinus, L. \& G. Chapuis, J. Appl. Cryst.2007, 41, 786-790. [3] Petricek, V., Eigner, V., Dusek, M. \& Cejchan, A. (2016) Z. Kristallogr. 231, 301-312. [4] de Wollf, P.M., Janssen T. \& Janner, A. (1981) Acta Cryst. A37, (1981) 625-636. [5] Petricek, V., Fuksa J. \& Dusek, M. (1981) Acta Cryst. A66, (2010) 649-655. [6] Perez-Mato, J.M., Ribeiro, J.L., Petricek, V.,\& Aroyo, M.I., (2012), J.Phys.: Condens. Matter 163201.

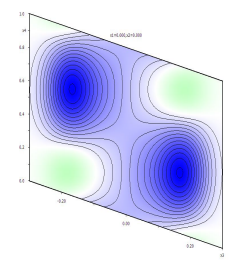

Figure 1

Acta Cryst. (2020). A76, a46 